doi: $10.2306 /$ scienceasia1513-1874.2012.38.147

\title{
Degradation of polycyclic aromatic hydrocarbons by newly isolated Curvularia sp. F18, Lentinus sp. S5, and Phanerochaete sp. T20
}

\author{
Kanokpan Juckpech $^{\mathrm{a}}$, Onruthai Pinyakong ${ }^{\mathrm{a}, \mathrm{b}}$, Panan Rerngsamran ${ }^{\mathrm{a}, \mathrm{b}, *}$ \\ a Bioremediation Research Unit, Department of Microbiology, Faculty of Science, Chulalongkorn University, \\ Bangkok 10330 Thailand \\ b National Centre of Excellence for Environmental and Hazardous Waste Management (NCE-EHWM), \\ Chulalongkorn University, Bangkok 10330 Thailand
}

*Corresponding author, e-mail: panan.r@chula.ac.th

Received 9 Nov 2011

Accepted 19 Apr 2012

\begin{abstract}
Three chromogenic substances with structures resembling those of polycyclic aromatic hydrocarbons (PAHs) were incorporated in culture medium in order to screen for fungi capable of degrading PAHs. Curvularia sp. F18, Lentinus sp. S5, and Phanerochaete sp. T20 were isolated and shown to have the ability to degrade both low- and highmolecular weight PAHs, with the most prominent degradation being observed with Phanerochaete sp. T20. Preliminary metabolite analysis of fluorene degradation by Phanerochaete sp. T20 using HPLC and GC-MS revealed that one of the early metabolites was 9-fluorenol, which is a less toxic substance. This fungus survived in $500 \mathrm{mg} / \mathrm{l}$ of PAH for at least 30 days. The fungus could degrade a mixture of four PAHs (25 mg/l each), resulting in the reduction of 97, 59, 39, and $47 \%$ of fluorene, phenanthrene, fluoranthene, and pyrene, respectively. This work demonstrates that Phanerochaete sp. T20 could be used to bioremediate environments contaminated with high concentrations and/or mixtures of PAHs.
\end{abstract}

KEYWORDS: biodegradation, bioremediation, fungi, mixed PAHs

\section{INTRODUCTION}

Polycyclic aromatic hydrocarbons (PAHs) are a group of environmental pollutants that are composed of carbon and hydrogen with fused benzene rings in linear, angular, and clustered arrangements. Based on the molecular weight of these hydrocarbons, PAHs can be classified into two broad groups: (i) the lowmolecular weight PAHs that contain 2-3 benzene rings, such as naphthalene, fluorene, and phenanthrene, and (ii) the high-molecular weight PAHs, such as fluoranthene, pyrene, and chrysene ${ }^{1}$. PAHs are generated as byproducts of incomplete combustion of organic substances, which are found in burnt fossil fuels, forest fires, volcano eruptions, and motor vehicle emissions, as well as in grilled and smoked foods ${ }^{2}$. PAHs can also be found as contaminants at industrial sites, especially those associated with petroleum or gas production and wood preserving processes $^{3,4}$. PAHs and their metabolites are reported to possess mutagenic and carcinogenic properties for humans and other animals ${ }^{5,6}$. Consequently, the US Environmental Protection Agency has listed some PAHs as priority pollutants ${ }^{1}$. Generally, the highmolecular-weight PAHs are less water-soluble and more recalcitrant to degradation than low-molecularweight PAHs ${ }^{7}$. Investigations of the content of PAHs found in several contaminated areas reveal that contamination is the result of a mixture of PAHs rather than a single type of contaminant ${ }^{4,8-10}$. Due to the long half life of PAHs and the human activities that cause the emissions of these contaminants into the environment every day, PAHs continuously increase and accumulate in the soil, water, and sediments and thus appropriate treatment is required to reduce the concentration and toxicity of these substances.

Chemical methods, such as chemical oxidation and liquid solvent extraction, and physical methods, such as incineration and microwave energy treatments, have been shown to have high levels of efficiency in remediating sites contaminated with PAHs ${ }^{11}$. However, these methods require complex technologies, have high treatment cost, tend to use excessive amounts of organic solvents, and may harm living organisms ${ }^{12}$. Bioremediation, a safe, environmentally friendly, and effective method, uses the ability of organisms, such as bacteria, fungi, algae, or plants, to reduce the concentrations of PAHs to an acceptable level by transforming them into less toxic forms or to completely mineralize them into 
$\mathrm{CO}_{2}{ }^{13}$. Fungi have advantages over other organisms in that they produce classes of enzymes, such as lignin peroxidase, manganese peroxidase, and laccase, that can interact with several types of PAHs with a fairly high degree of non-specific activity ${ }^{14}$. They also have other enzymatic systems, such as cytochrome P450 monooxygenase and epoxide hydrolase that oxidize PAHs ${ }^{15,16}$. Fungi are also tolerant to high concentrations of recalcitrant compounds and are able to flourish in extreme conditions, such as at high temperatures and under low $\mathrm{pH}$ conditions. In addition, the fact that fungi form large, branching mycelia makes it possible for them to grow and distribute through a solid matrix to degrade PAHs within contaminated areas (in situ) by virtue of secreting extracellular enzymes or by sequestration of PAHs ${ }^{17-19}$. Fungi can also degrade PAHs under microaerobic and very-lowoxygen conditions ${ }^{20}$. In addition to biodegradation and mineralization of the PAHs, fungi adsorb PAHs onto their hydrophobic cell wall ${ }^{21}$ and/or store them in vacuoles or other organelles inside the cells ${ }^{22,23}$. In combination, all of these mechanisms lead to the reduction of PAHs in the environment. Several reports have demonstrated that fungi, such as Phanerochaete chrysosporium, Cunninghamella elegans, Trametes versicolor, Bjerkandera adusta, and Pleurotus ostreatus, play an important role in the degradation of a wide variety of xenobiotic compounds, including PAHs ${ }^{18}$. Most of the current research in the field has studied the ability of a specific fungus to degrade a particular PAH compound ${ }^{24-27}$. However, contaminated sites are commonly contaminated with a mixture of PAHs. Therefore, the objective of this study was to screen for fungi that could degrade a mixture of four PAHs, including fluorene and phenanthrene, and fluoranthene and pyrene, as representatives of low- and highmolecular weight PAHs, respectively. These fungi have the potential to be used for in situ bioremediation at environmental sites where contamination is caused by several types of PAHs, a more common scenario.

\section{MATERIALS AND METHODS}

\section{Polycyclic aromatic hydrocarbons (PAHs)}

Fluorene was obtained from the Wako Pure Chemical Industries Co. (Japan). Fluoranthene was obtained from the Kanto Chemical (Japan). Phenanthrene, pyrene, benomyl, and the three chromogenic $\mathrm{PAH}-$ like substances (guaiacol, azureB, and phenol red) were obtained from the Sigma-Aldrich Co. (USA), 9-fluorenone was obtained from Nacalai Tesque Co. (Japan), and 9-fluorenol was obtained from the TCI Co. (Japan). All chemicals were of analytical grade.

\section{Media}

Two percent malt extract agar (MEA), used for the preliminary isolation of fungi, contained (per litre): $20 \mathrm{~g}$ malt extract, $5 \mathrm{~g}$ peptone, $20 \mathrm{~g}$ glucose, $15 \mathrm{~g}$ agar, $3 \mathrm{mg}$ benomyl, and $50 \mathrm{mg}$ chloramphenicol ${ }^{28}$.

Minimal medium (MM), used for peroxidase enzyme screening, contained (per litre): $0.5 \mathrm{~g} \mathrm{KH}_{2} \mathrm{PO}_{4}$, $0.5 \mathrm{~g} \mathrm{MnSO}_{4}, 0.1 \mathrm{~g} \mathrm{NH}_{4} \mathrm{NO}_{3}, 18 \mathrm{~g}$ agar, and $200 \mathrm{ml}$ of trace elements solution containing the following reagents (per litre): $5 \mathrm{~g} \mathrm{Na}_{2}$ EDTA, $0.5 \mathrm{~g} \mathrm{FeCl}_{3}, 0.05 \mathrm{~g}$ $\mathrm{ZnCl}_{2}, 0.01 \mathrm{~g} \mathrm{CuCl}_{2}, 0.01 \mathrm{~g} \mathrm{CoCl}_{2} \cdot 6 \mathrm{H}_{2} \mathrm{O}, 0.01 \mathrm{~g}$ $\mathrm{H}_{3} \mathrm{BO}_{3}$, and $1.6 \mathrm{~g} \mathrm{MnCl}_{2}{ }^{25}$.

Modified GPY (mGPY), used for the preparation of fungal inocula, contained (per litre): $10 \mathrm{~g}$ glucose, $3 \mathrm{~g}$ peptone, $2 \mathrm{~g}$ yeast extract, $1 \mathrm{~g} \mathrm{K \textrm {KH } _ { 2 }} \mathrm{PO}_{4}, 1 \mathrm{~g}$ $\mathrm{MgSO}_{4} \cdot 7 \mathrm{H}_{2} \mathrm{O}$, and $0.4 \mathrm{~g}$ Na-tartrate ${ }^{25}$.

$\mathrm{N}$-limited medium, used for the biodegradation experiments, contained (per litre): $10 \mathrm{~g}$ glucose, $0.1 \mathrm{~g} \mathrm{NH}_{4} \mathrm{NO}_{3}, 1 \mathrm{~g} \mathrm{KH}_{2} \mathrm{PO}_{4}, 1 \mathrm{~g} \mathrm{MgSO} \mathrm{M}_{4} \cdot 7 \mathrm{H}_{2} \mathrm{O}$, $0.01 \mathrm{~g} \mathrm{FeSO}_{4} \cdot 7 \mathrm{H}_{2} \mathrm{O}, 0.01 \mathrm{~g} \mathrm{ZnSO}_{4} \cdot 7 \mathrm{H}_{2} \mathrm{O}, 0.001 \mathrm{~g}$ $\mathrm{MnSO}_{4}$, and $0.001 \mathrm{~g} \mathrm{CuSO}_{4} \cdot 5 \mathrm{H}_{2} \mathrm{O}^{29}$.

\section{Fungal isolation}

Wood-rot fungi or mushrooms growing on rotten wood and soil contaminated with petroleum oil were collected from five provinces in Thailand (Bangkok, Chonburi, Nakhon Pathom, Phatthalung, and Rayong). Pieces of the inner tissue of the mushroom or rotten wood fungi were placed on MEA media containing $3 \mathrm{mg} / \mathrm{l}$ benomyl and $50 \mathrm{mg} / \mathrm{l}$ chloramphenicol to inhibit fast-growing fungi and bacteria, respectively. Fungi from soil samples were isolated using the soil dilution plate technique on MEA media bearing benomyl and chloramphenicol. All plates were incubated at room temperature for 5-7 days. The fungi were isolated as pure cultures using the same media. The purified isolates were kept as stock cultures at $4{ }^{\circ} \mathrm{C}$ on MEA slants until used.

\section{Screening for potential fungi using chromogenic substances}

$0.1 \%$ Guaiacol, $0.1 \%$ azureB, and $0.0025 \%$ phenol red were used for the screening of potential fungi that could produce peroxidase and laccase enzymes ${ }^{30-33}$. Three 7-mm agar plugs containing fungal mycelia from 7-day-old cultures on MEA plates were placed on an MM plate that contained each chromogenic substance. All MM plates were incubated in the dark at room temperature for 3 days. The fungi that were able to change these chromogenic substances, as determined by visual appearance of a different coloured halo around the fungal colony, were selected 
for further study.

Fungal identification was performed by ITS1 - 5.8 RNA - ITS2 DNA sequence identity using standard conditions and primers ITS1 and ITS4 or ITS1-F and ITS4 ${ }^{34}$. Direct sequencing of both strands of each purified amplicon was commercially performed by 1st BASE DNA Sequencing Service (Malaysia). The consensus nucleotide sequences were compared to those available in the GenBank database using the BLASTn algorithm.

\section{Degradation of PAHs in liquid medium and metabolite analysis}

Fungi that exhibited positive results from the screening step were prepared for inocula in $100 \mathrm{ml}$ of mGPY liquid medium at $30^{\circ} \mathrm{C}$ with shaking at $120 \mathrm{rpm}$ for 5 days. Mycelia were harvested by centrifugation at $1120 \mathrm{~g}$ for $15 \mathrm{~min}$ and washed twice with $0.85 \%(\mathrm{w} / \mathrm{v})$ $\mathrm{NaCl}$. Three grams of fresh mycelia were added into $30 \mathrm{ml}$ of N-limited medium in 125-ml Erlenmeyer flasks containing three glass marbles. Each PAH $(100 \mathrm{mg} / \mathrm{l})$ was added to these cultures and shaken at $120 \mathrm{rpm}$ in the dark at $30^{\circ} \mathrm{C}$. For the control experiment, the flasks containing fungal mycelia were autoclaved at $121^{\circ} \mathrm{C}$ for $15 \mathrm{~min}$ prior to adding the PAH. Samples were collected 15 days after incubation.

PAHs and their metabolites were extracted from $5 \mathrm{ml}$ of samples using ethyl acetate, as previously described $^{24}$, and analysed by HPLC. HPLC analysis was performed with a liquid chromatograph system (Shimadzu) equipped with an LC-3A pump, an SPD-2A UV-Vis detector and a C-RIA recorder. The separation column was $4.6 \times 150 \mathrm{~mm}$ (Inersil ODS-3) and the mobile phase was methanol:water $(80: 30(\mathrm{v} / \mathrm{v}))$ at a flow rate of $1 \mathrm{ml} / \mathrm{min}$. The reduction of each PAH was calculated as $\left(1-A_{\mathrm{T}} / A_{\mathrm{C}}\right)$, where $A_{\mathrm{C}}$ is the area under the peak of the substrate from the control set and $A_{\mathrm{T}}$ is the area under the peak of the substrate from the test set. Prior to extraction step of some experiments, $100 \mathrm{mg} / \mathrm{l}$ of pyrene was added as an internal standard to monitor the extraction efficiency.

For metabolite analysis, the metabolites were collected at the optimum production time. These metabolites were acidified with hydrochloric acid to pH 2-3 and extracted by ethyl acetate, evaporated, resuspended in ethanol, and evaporated a second time ${ }^{24}$. The precipitate was resuspended in methanol and these were analysed for the presence of PAHs and metabolites by HPLC and GC-MS. HPLC analyses were performed as described above. Samples for GC-MS analyses were analysed using gas chromatography with time of flight mass spectrometry (Pegasus
III, LECO). The GC used a $50 \mathrm{~m}$ long HP-5 column of $320 \mu \mathrm{m}$ in diameter and was coated to a $0.25-\mu \mathrm{m}$ film thickness with 5\% phenyl-methyl-syloxane.

\section{Survival of selected fungi in different concentration of PAHs}

Three grams of fresh mycelia of each selected fungus were inoculated into $\mathrm{N}$-limited medium supplemented with each single $\mathrm{PAH}$ at 25, 50, 100, 300, and $500 \mathrm{mg} / \mathrm{l}$ and was incubated in the dark at $30^{\circ} \mathrm{C}$ for 30 days. To test for the survival of the fungus, $100 \mu \mathrm{l}$ of fungal culture was dropped on to MEA medium. The plate was incubated for 7 days and fungal growth was observed.

\section{Ability of fungi to grow on solid medium containing mixed PAHs}

The four PAHs, including fluorene, phenanthrene, fluoranthene, and pyrene, were incorporated into MM agar plates at a concentration of $25 \mathrm{mg} / \mathrm{l}$ each. Three 7-mm agar plugs containing fungal mycelia from 7day-old cultures grown on MEA plates were placed on each of the MM plates. All plates were incubated in the dark at room temperature for 10 days.

\section{Degradation of mixed PAHs in liquid medium}

Each fungal inoculum was prepared and $3 \mathrm{mg}$ of fresh mycelia were added into $30 \mathrm{ml}$ of $\mathrm{N}$-limited medium in $125 \mathrm{ml}$ Erlenmeyer flasks containing three glass marbles. A mixture of the four PAHs $(25 \mathrm{mg} / \mathrm{l} \mathrm{each})$ was added to each flask. All flasks were shaken at $120 \mathrm{rpm}$ in the dark at $30^{\circ} \mathrm{C}$. Flasks containing fungal mycelia that were autoclaved at $121^{\circ} \mathrm{C}$ for 15 min prior to the addition of the PAHs were used as controls. Samples were collected at 15 days after incubation, whereupon PAHs were extracted and analysed as previously described ${ }^{24}$. All data are presented as the mean value derived from duplicate samples.

\section{RESULTS}

\section{Fungal isolation and screening}

From the initial 55 fungal isolates, 47 isolates (85.5\%) were unable to change the colour of any of the three indicator compounds. However, eight isolates $(14.5 \%)$ were able to change at least one of the three chromogenic substances. Among the latter, three isolates consisting of $\mathrm{F} 18, \mathrm{~S} 5$, and $\mathrm{T} 20$ gave the widest zone of colour changes and, therefore, were selected for further characterization because the colour changes of these substances have been reported to be related to the production of peroxidase and laccase enzymes, which are responsible for the degradation of $\mathrm{PAHs}^{30-33}$. 
Nucleotide sequencing of the ITS regions of the rRNA genes (ITS1 - 5.8S RNA - ITS2) was performed for the F18, S5, and T20 isolates followed by BLASTn searches to identify close relatives (high sequence identity) in the GenBank database. The F18 isolate had $100 \%$ nucleotide sequence identity to the sequence designated as Curvularia sp. F SMR-2011 (HQ909079). Isolates S5 and T20 showed 98\% and 93\% sequence identity to those designated as Lentinus squarrosulus strain 7-4-2 (GU001951) and Phanerochaete sp. ATT215 (HQ607891), respectively. In this study, we refer to these isolates as Curvularia sp. F18, Lentinus sp. S5 and Phanerochaete sp. T20. The consensus ITS nucleotide sequences for isolates F18, S5, and T20 have been submitted to the GenBank database under accession numbers JN253597, JN253598, and JN253599, respectively.

\section{Degradation of single PAH in liquid medium}

These three fungi were tested for their ability to degrade the four representative PAHs of fluorene, phenanthrene, fluoranthene, and pyrene in nitrogenlimited liquid media $(100 \mathrm{mg} / \mathrm{l})$. After analysis of the metabolites by HPLC, the areas under the peaks were compared to that of the control set using the killed fungus. The reduction for each PAH was calculated using the aforementioned formula. Curvularia sp. F18 was found to be unable to degrade fluoranthene, phenanthrene, and pyrene, as the substrate peaks were still present (data not shown). However, Curvularia sp. F18 was able to degrade fluorene, as it showed a $90 \%$ reduction in the initial level added after 15 days of incubation. In addition, the analysis of Curvularia sp. F18 showed the appearance of four major new peaks (Fig. 1) that are assumed to be intermediate metabolites. Lentinus sp. S5 was unable to degrade fluoranthene under these conditions (data not shown) but degraded fluorene, phenanthrene, and pyrene at 60,86 , and $85 \%$ relative to the control cultures, respectively, (Fig. 2). Finally, Phanerochaete sp. T20 was able to degrade $83 \%$ of fluorene, $87 \%$ of phenanthrene, and $31 \%$ of fluoranthene (Fig. 3) but was unable to degrade pyrene (data not shown).

\section{Metabolite analysis}

Although the results indicate that Lentinus sp. S5 and Phanerochaete sp. T20 are potentially good candidates for PAHs degradation, isolate S5 exhibited a slow growth rate. Thus we selected Phanerochaete sp. T20 for further characterization. This fungus was also closely related to $P$. chrysosporium, which is the most reported PAH-degrading fungus ${ }^{35-38}$. In order to determine the preliminary PAH degradation
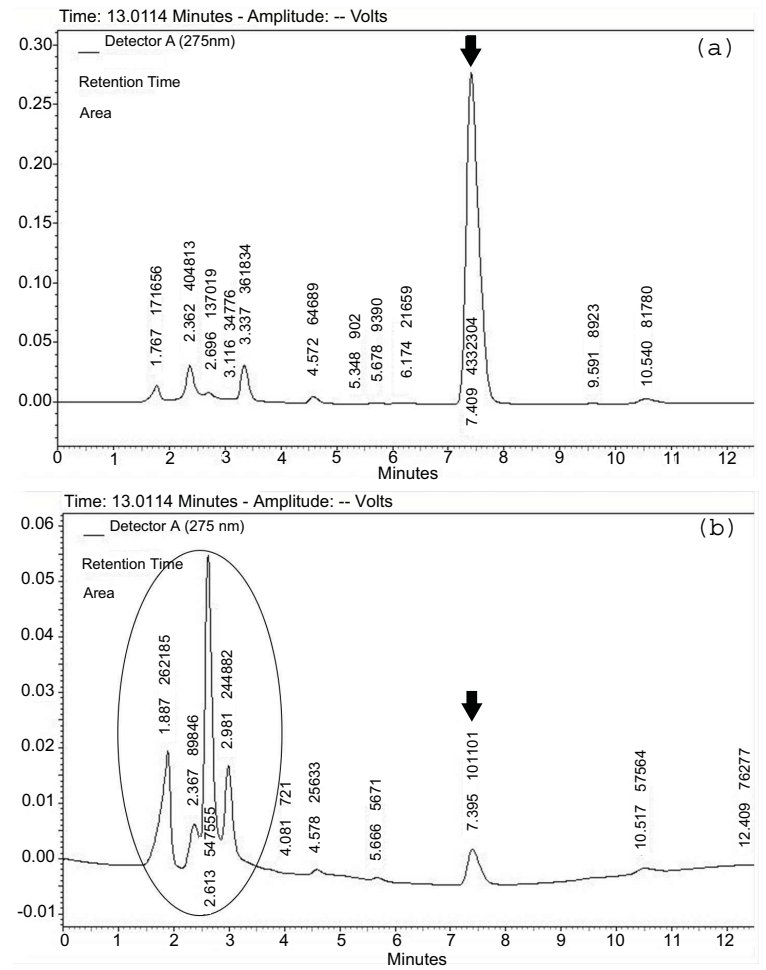

Fig. 1 HPLC chromatograms of fluorene degradation by Curvularia sp. F18. Representative HPLC chromatograms from a 15-day-old culture of (a) autoclaved and (b) live mycelia of Curvularia sp. F18 grown in N-limited media containing fluorene at $100 \mathrm{mg} / \mathrm{l}$. Arrows indicate the peaks of fluorene. A circle specifies the intermediate peaks.

pathway used by Phanerochaete sp. T20, we determined the early metabolites that arose from the degradation of fluorene. The Phanerochaete sp. T20 fungus was grown in $\mathrm{N}$-limited medium supplemented with $100 \mathrm{mg} / \mathrm{l}$ of fluorene. The first intermediate peak was observed on the third day of incubation and was extracted, purified, and analysed by HPLC and GC-MS. In each case, 9-fluorenol and 9-fluorenone were used as external standards because these products have been reported to be the first metabolites of fluorene degradation in most white rot fungi ${ }^{39}$. The HPLC analysis also revealed that the purified intermediate had the same retention time $(\mathrm{RT}=$ 0.71) as 9-fluorenol (Fig. 4), while GC-MS analysis revealed that the purified intermediate gave $\mathrm{m} / z$ ratio values of $63,76,91,126,152$, and 181 , which were the same as those for 9-fluorenol. Taken together, the results from HPLC and GC-MS methods strongly suggested that the first observed intermediate from fluorene degradation by Phanerochaete sp. T20 was likely to be 9-fluorenol. 

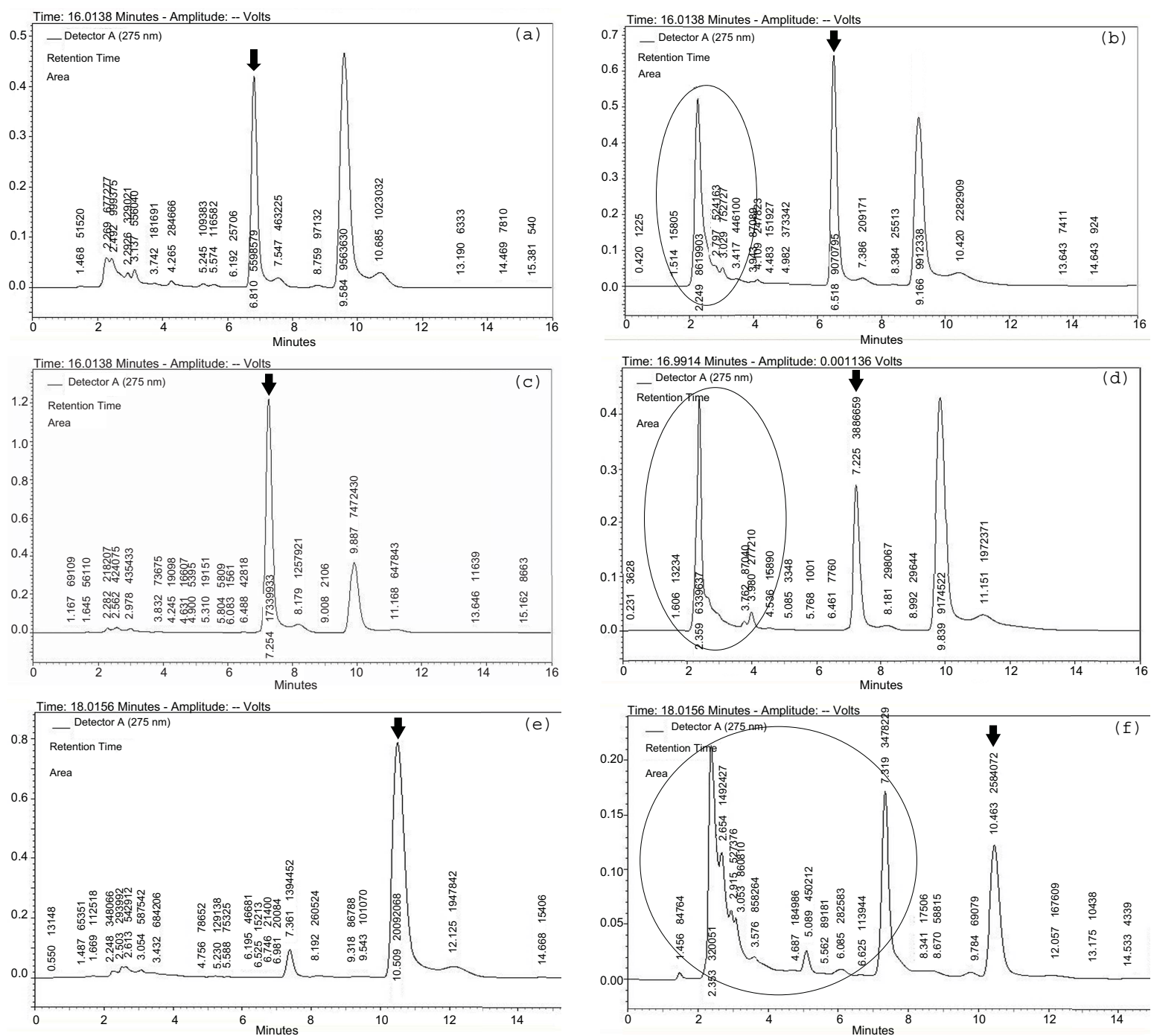

Fig. 2 HPLC chromatograms of fluorene, phenanthrene, and pyrene degradation by Lentinus sp. S5. Representative HPLC chromatograms from a 15-day-old culture of ( $a, c, e)$ autoclaved and (b, d, f) live mycelia of Lentinus sp. S5 grown in $\mathrm{N}$-limited media containing (a, b) fluorene, $(c, d)$ phenanthrene, and $(e, f)$ pyrene at $100 \mathrm{mg} / \mathrm{l}$. Arrows indicate the peaks of fluorene. Circles specify the intermediate peaks. The internal loading standard of pyrene is found to the right of the substrate peaks in the fluorene and phenanthrene degradations.

\section{Survival of Phanerochaete sp. T20 at various concentrations of PAHs}

To investigate the ability of this fungus to survive under different concentrations of PAHs, fresh mycelia from mGPY medium were cultured in media containing different concentrations $(25,50,100,300$, or $500 \mathrm{mg} / \mathrm{l}$ ) of fluorene, phenanthrene, fluoranthene, or pyrene. After 30 days, survival of the fungus was investigated by dropping the fungal culture onto an MEA plate. Growth of Phanerochaete sp. T20 was observed on MEA plates from all five tested concentrations of all four evaluated PAHs (data not shown). The fungus was able to survive, at least to some extent, in all four tested PAHs at concentration of up to $500 \mathrm{mg} / \mathrm{l}$ for at least 30 days.

\section{Testing the ability of Phanerochaete sp. T20 to grow on solid medium containing mixed PAHs and to degrade mixed PAHs in liquid medium}

To further test for the potential of Phanerochaete sp. T20 to degrade a mixture of PAHs, this isolate was evaluated for its ability to grow on MM agar medium containing an equal concentration of fluorene, phenanthrene, fluoranthene, and pyrene as the sole carbon 

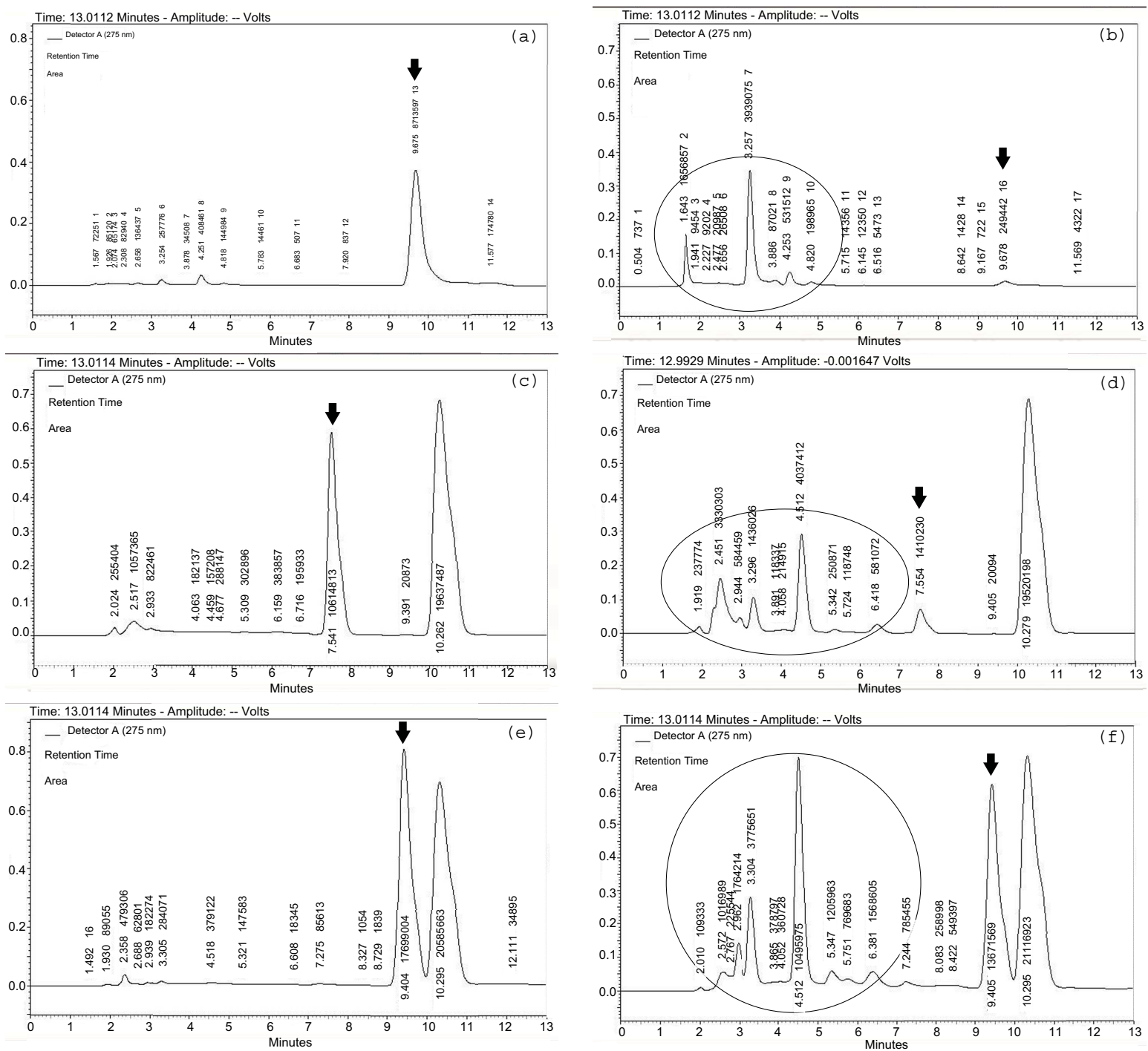

Fig. 3 HPLC chromatograms of fluorene, phenanthrene, and fluoranthene degradations by Phanerochaete sp. T20. Representative HPLC chromatograms from a 15-day-old culture of (a, c, e) autoclaved and (b, d, f) live mycelia of Phanerochaete sp. T20 grown in N-limited media containing $(\mathrm{a}, \mathrm{b})$ fluorene, $(\mathrm{c}, \mathrm{d})$ phenanthrene, and $(\mathrm{e}, \mathrm{f})$ fluoranthene at $100 \mathrm{mg} / \mathrm{l}$. Arrows indicate the peaks of fluorene. Circles specify the intermediate peaks. The internal loading standard of pyrene is located to the right of the substrate peak.

sources. Clear growth was observed on this medium, although with a slower growth rate compared to its growth on MEA medium alone (data not shown). This observation serves as a preliminary indication that this fungus should be able to use or degrade these mixed PAHs, therefore, we evaluated its ability to degrade mixed PAHs (25 mg/l each) in liquid medium. After 15 days in culture, PAHs and their metabolites were extracted from the culture media, analysed by HPLC and the results were compared with those obtained from the control cultures (autoclaved mycelia). A decrease in the area of the substrate peaks and the appearance of new peaks (assumed metabolites) associated with the live mycelia cultures, but not in the autoclaved controls, were indicators of the ability of the fungus to degrade multiple PAHs (Fig. 5). These results indicated that Phanerochaete sp. T20 could degrade a mixture of four different PAHs with initial concentrations of $25 \mathrm{mg} / \mathrm{l}$ each. Next, we calculated the reduction in the level of each PAH relative to the level in the corresponding control (autoclaved mycelia), and the results revealed that Phanerochaete 

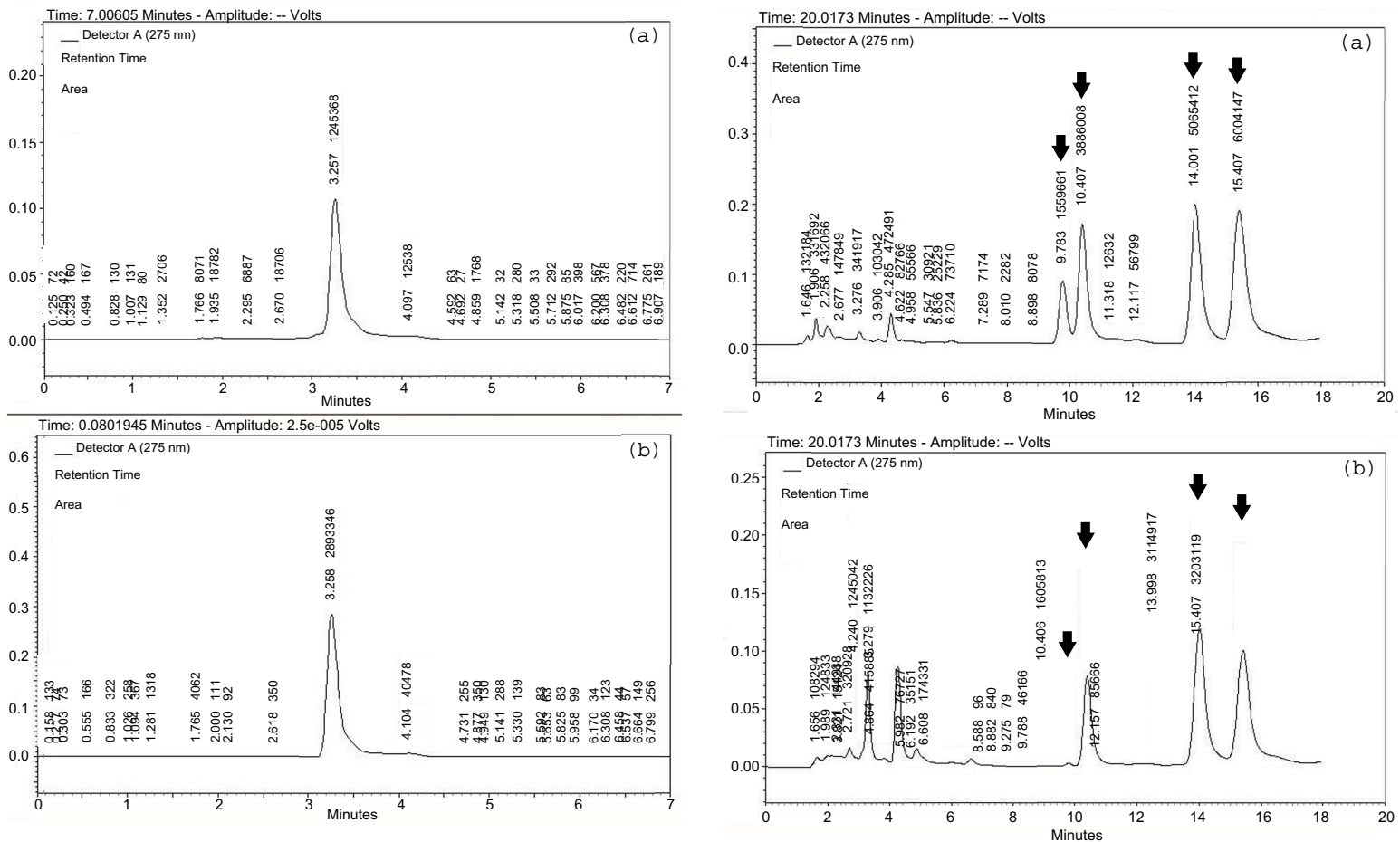

Fig. 4 HPLC chromatograms of purified intermediate and 9fluorenol. Representative HPLC chromatograms illustrating (a) the purity of the purified intermediate compound from a three-day-old culture of Phanerochaete sp. T20 grown in $\mathrm{N}$-limited media containing fluorene at $100 \mathrm{mg} / \mathrm{l}$ and (b) 9fluorenol.

sp. T20 was able to remove fluorene, phenanthrene, pyrene, and fluoranthene by $97,59,47$, and $39 \%$, respectively, (Fig. 5). Thus this fungus has the potential to degrade and remove a mixture of at least four PAHs (fluorene, phenanthrene, fluoranthene, and pyrene).

\section{DISCUSSION}

The white-rot fungi are a group of organisms capable of degrading a wide variety of environmental pollutants, including PAHs, due to the production of extracellular and relatively nonspecific (for the substrate) ligninolytic enzymes, including lignin peroxidase, manganese peroxidase, and laccase ${ }^{13}$. In this study, we initially isolated fungi that had the potential to degrade PAHs by screening for the ability of these isolates to degrade the chromogenic substrates phenol red, guaiacol, and azureB, which have structures that resemble PAHs. We used these substrates as indicators for the potential production of extracellular peroxidase and/or laccase enzymes. Two white-rot fungi, which were identified as Lentinus sp. S5 and Phanerochaete sp. T20, and one Ascomycetes fungus, Curvularia sp. F18, were isolated using this criterion. These fungi

Fig. 5 HPLC chromatograms of mixed-PAHs degradation by Phanerochaete sp. T20. Representative HPLC chromatograms from a 15-day-old culture of (a) autoclaved and (b) live mycelia of Phanerochaete sp. T20 grown in $\mathrm{N}$-limited media containing fluorene, phenanthrene, fluoranthene, and pyrene at $25 \mathrm{mg} / \mathrm{l}$ each. The retention time of fluorene, phenanthrene, fluoranthene, and pyrene were 9.6, 10.3, 13.9, and $15.3 \mathrm{~min}$, respectively. Arrows indicate the peaks of these four substrates.

were then tested for the ability to degrade two lowmolecular weight PAHs (phenanthrene and fluorene) and two high-molecular weight PAHs (fluoranthene and pyrene), both alone and together. All three fungal isolates were found to be good candidates for PAH bioremediation, showing good degradation of the lowmolecular weight PAHs. In addition, S5 and T20 also showed good and moderate degradation of the high-molecular weight pyrene and fluoranthene, respectively. Further characterization of Phanerochaete sp. T20 revealed that it can withstand and survive in high concentrations of PAHs (at least up to $500 \mathrm{mg} / \mathrm{l}$ for 30 days) and that the degradation of fluorene produced a less toxic compound, 9-fluorenol, as the major early intermediate. In addition, this strain showed the potential ability to degrade a mixture of all four of these PAHs.

Although fungi in the genus Curvularia have been reported to be able to degrade tricyclic PAHs, such 
as phenanthrene and anthracene ${ }^{40,41}$, the degradation of fluorene (which has a more complex structure) by fungi in this genus had never been demonstrated prior to this study. Our results indicate that, among the four PAHs tested, Curvularia sp. F18 could only degrade fluorene, a tricyclic PAH with a five-membered ring, at high efficiency. Although the intermediate metabolites of fluorene were not investigated in this study, several previous reports have shown that for non-white-rot fungi fluorene and a number of other PAHs are generally metabolized by cytochrome P450 monooxygenase and epoxide hydrolase to form transdihydrodiols ${ }^{39}$. Due to its robust ability to degrade fluorene, Curvularia sp. F18 should be further investigated for its potential use in situ bioremediation where high levels of fluorene contamination are present.

Lentinus sp. S5 is a member of the white-rot Basidiomycetes fungi. The fungi in this genus have been reported to degrade several types of PAHs using both extracellular ligninolytic enzymes and intracellular P450 monooxygenase systems ${ }^{42,43}$. In this study, Lentinus sp. S5 was capable of degrading phenanthrene, fluorene, and pyrene. However, it could not degrade fluoranthene. The chromatograms from the phenanthrene, fluorene, and pyrene degradations by Lentinus sp. S5 (Fig. 2) revealed new peaks with the same retention times for all three PAHs, indicating that Lentinus sp. S5 used the same pathway or the same mechanism to degrade these PAHs, giving rise to the same intermediate metabolites. This possibility remains to be confirmed. However, if this hypothesis is correct, these metabolites would probably be the PAH-quinone compounds, which, in general, are the products of PAHs degradation by white rot fungi including Lentinus via the ligninolytic and laccase enzymatic reactions ${ }^{42}$. In the case of complete mineralization, this $\mathrm{PAH}$-quinone will pass the ring fission process and produce $\mathrm{CO}_{2}$ as the final product ${ }^{44}$.

Phanerochaete sp. T20 is a white-rot Basidiomycetes fungus that belongs to the same genus as the well-known PAHs degrader, P. chrysosporium $^{35,45}$. In the degradation of each $\mathrm{PAH}$ alone, Phanerochaete sp. T20 appeared to be capable of degrading fluorene, phenanthrene, and fluoranthene; and several intermediate peaks were seen in the chromatograms of the degradation. However, it could not degrade pyrene, which is a fused tetracyclic aromatic high-molecular weight hydrocarbon. In contrast, when Phanerochaete sp. T20 was grown in N-limiting medium containing all four PAHs, it was able to degrade pyrene, as well as fluorene, phenanthrene, and fluoranthene, and at a higher level than fluoranthene alone. The apparent degradation of pyrene in the presence of other easily degradable substrates would probably result from the interactions between substrates that subsequently enhance the degradation of compounds that are more difficult to degrade, such as pyrene ${ }^{46}$. Synergistic effect between PAHs and cometabolism were also proposed for this feature ${ }^{47}$. In addition to its ability to degrade a mixture of low- and high-molecular weight PAHs, Phanerochaete sp. T20 was able to grow and survive (at least to some extent) at relatively high concentrations of PAHs $(500 \mathrm{mg} / \mathrm{l})$ for at least 30 days. These results indicate a notable PAH degradation efficiency of Phanerochaete sp. T20 and suggest that this fungus might be useful for in situ bioremediation at sites where mixed and/or high concentrations of PAHs are a problem.

The early metabolites of fluorene degradation were further investigated to preliminarily determine the degradation pathway of Phanerochaete sp. T20. The results from HPLC and GC-MS indicated that this metabolite was most probably 9-fluorenol, which is a less mutagenic compound than fluorene ${ }^{39}$. This observation is considered to be an indicator for the first step of fluorene detoxification ${ }^{11}$. Thus Phanerochaete sp. T20 likely degrades fluorene under nonligninolytic conditions such that it degrades fluorene via cytochrome $\mathrm{P} 450$ monooxygenase, as is frequently found with $P$. chrysosporium and other white-rot fungi ${ }^{39,48,49}$.

The three PAH-degrading fungi that were isolated in this study can potentially be used for bioremediation. Curvularia sp. F18 may be suitable for conditions where the contamination is fluorene, while Lentinus sp. S5 and Phanerochaete sp. T20 may be suitable for contamination sites where more than one type of PAHs is the main concern. This study reports on the preliminary isolation and investigation of three PAHdegrading fungi. However, further detailed studies are required to investigate the degradation pathways and the degradation products of these useful fungi.

Acknowledgements: This study was financially supported by a grant for the development of new faculty at Chulalongkorn University; the Thai Government Stimulus Package 2 (TKK2555), under the Project for Establishment of Comprehensive Centre for Innovative food, Health Products and Agriculture; and a CU graduate school thesis grant.

\section{REFERENCES}

1. Wilson SC, Jones KC (1993) Bioremediation of soil contaminated with polynuclear aromatic hydrocarbons (PAHs): A review. Environ Pollut 81, 229-49.

2. Clemente AR, Anazawa TA, Durrant LR (2001) Biodegradation of polycyclic aromatic hydrocarbons by soil fungi. Braz J Microbiol 32, 255-61. 
3. Muncnerova D, Augustin J (1994) Fungal metabolism and detoxification of polycyclic aromatic-hydrocarbons - A review. Bioresource Tech 48, 97-106.

4. Thavamani P, Megharaj M, Krishnamurti GS, McFarland R, Naidu R (2011) Finger printing of mixed contaminants from former manufactured gas plant (MGP) site soils: Implications to bioremediation. Environ Int 37, 184-9.

5. Arun A, Eyini M (2011) Comparative studies on lignin and polycyclic aromatic hydrocarbons degradation by basidiomycetes fungi. Bioresource Tech 102, 8063-70.

6. Samanta SK, Singh OV, Jain RK (2002) Polycyclic aromatic hydrocarbons: Environmental pollution and bioremediation. Trends Biotechnol 20, 243-8.

7. Shuttleworth KL, Cerniglia CE (1995) Environmental aspects of PAH biodegradation. Appl Biochem Biotechnol 54, 291-302.

8. Norramit P, Cheevaporn V, Itoh N, Tanaka K (2005) Characterization and carcinogenic risk assessment of polycyclic aromatic hydrocarbons in the respirable fraction of airborne particles in the Bangkok metropolitan area. J Health Sci 51, 437-46.

9. Oanh NTK, Reutergardh LB, Dung NT, Yu MH, Yao WX, Co HX (2000) Polycyclic aromatic hydrocarbons in the airborne particulate matter at a location 40 $\mathrm{km}$ north of Bangkok, Thailand. Atmos Environ 34, 4557-63.

10. Wattayakorn G (2003) Polycyclic aromatic hydrocarbons in the Chao Phraya Estuary, Thailand. J Sci Res Chula Univ 28, 17-27.

11. Singh H (2006) Fungal metabolism of polycyclic aromatic hydrocarbons. In: Singh H (ed) Mycoremediation: Fungal Bioremediation. John Wiley \& Sons, New Jersey, pp 283-356.

12. Blanco EV, Mahía PL, Lorenzo SM, Rodríguez DP, Fernández EF (2000) Optimization of microwaveassisted extraction of hydrocarbons in marine sediments: Comparison with the Soxhlet extraction method. Fresen J Anal Chem 366, 283-8.

13. Pointing SB (2001) Feasibility of bioremediation by white-rot fungi. Appl Microbiol Biotechnol 57, 20-33.

14. Cerniglia CE, Sutherland JB (2001) Bioremediation of polycyclic aromatic hydrocarbons by ligninolytic and non-ligninolytic fungi. In: Gadd GM (ed) Fungi in Bioremediation. Cambridge Univ Press, Cambridge, pp 136-87.

15. Bezalel L, Hadar Y, Fu PP, Freeman JP, Cerniglia CE (1996) Initial oxidation products in the metabolism of pyrene, anthracene, fluorene, and dibenzothiophene by the white rot fungus Pleurotus ostreatus. Appl Environ Microbiol 62, 2554-9.

16. Syed K, Doddapaneni H, Subramanian V, Lam YW, Yadav JS (2010) Genome-to-function characterization of novel fungal P450 monooxygenases oxidizing polycyclic aromatic hydrocarbons (PAHs). Biochem Biophys Res Comm 399, 492-7.

17. Barr DP, Aust SD (1994) Pollutant degradation by white rot fungi. Rev Environ Contam Toxicol 138, 49-72.

18. Cerniglia CE (1997) Fungal metabolism of polycyclic aromatic hydrocarbons: Past, present and future applications in bioremediation. J Ind Microbiol Biotechnol 19, 324-33.

19. Reddy CA, Mathew Z (2001) Bioremediation potential of white rot fungi. In: Gadd GM (ed) Fungi in Bioremediation. Cambridge Univ Press, Cambridge, pp.

20. Silva IS, Grossman M, Durrant LR (2009) Degradation of polycyclic aromatic hydrocarbons (2-7 rings) under microaerobic and very-low-oxygen conditions by soil fungi. Int Biodeter Biodegr 63, 224-9.

21. Tekere M, Read JS, Mattiasson B (2005) Polycyclic aromatic hydrocarbon biodegradation in extracellular fluids and static batch cultures of selected sub-tropical white rot fungi. J Biotechnol 115, 367-77.

22. Verdin A, Lounes-Hadj Sahraoui A, Newsam R, Robinson G, Durand R (2005) Polycyclic aromatic hydrocarbons storage by Fusarium solani in intracellular lipid vesicles. Environ Pollut 133, 283-91.

23. Gao Y, Cao X, Kang F, Cheng Z (2011) PAHs pass through the cell wall and partition into organelles of arbuscular mycorrhizal roots of ryegrass. $J$ Environ Qual 40, 653-6.

24. Bezalel L, Hadar Y, Fu PP, Freeman JP, Cerniglia CE (1996) Metabolism of phenanthrene by the white rot fungus Pleurotus ostreatus. Appl Environ Microbiol 62, 2547-53.

25. Chupungars K, Rerngsamran P, Thaniyavarn S (2009) Polycyclic aromatic hydrocarbons degradation by Agrocybe sp. CU-43 and its fluorene transformation. Int Biodeter Biodegr 63, 93-9.

26. Dhawale SW, Dhawale SS, Dean-Ross D (1992) Degradation of phenanthrene by Phanerochaete chrysosporium occurs under ligninolytic as well as nonligninolytic conditions. Appl Environ Microbiol 58, 3000-6.

27. Li P, et al (2005) Biodegradation of pyrene and phenanthrene in soil using immobilized fungi Fusarium sp. Bull Environ Contam Toxicol 75, 443-50.

28. Chiocchio V, Venedikian N, Martinez AE, Menendez A, Ocampo JA, Godeas A (2000) Effect of the fungicide benomyl on spore germination and hyphal length of the arbuscular mycorrhizal fungus Glomus mosseae. Int Microbiol 3, 173-5.

29. Kirk CJ, Verrinder TR, Hems DA (1978) The influence of extracellular calcium concentration on the vasopressin-stimulated incorporation of inorganic phosphate into phosphatidylinositol in hepatocyte suspensions. Biochem Soc Trans 6, 1031-3.

30. Archibald FS (1992) A new assay for lignin-type peroxidases employing the dye azure B. Appl Environ Microbiol 58, 3110-6.

31. Boominathan K, Dass SB, Randall TA, Kelley RL, Reddy CA (1990) Lignin peroxidase-negative mutant of the white-rot basidiomycete Phanerochaete chrysosporium. J Bacteriol 172, 260-5. 
32. Kiiskinen LL, Ratto M, Kruus K (2004) Screening for novel laccase-producing microbes. J Appl Microbiol 97, 640-6.

33. Pangallo D, Simonovicova A, Chovanova K, Ferianc P (2007) Wooden art objects and the museum environment: Identification and biodegradative characteristics of isolated microflora. Lett Appl Microbiol 45, 87-94.

34. Gardes M, Bruns TD (1993) ITS primers with enhanced specificity for Basidiomycetes: Application to the identification of mycorrhizae and rusts. Mol Ecol 2, $113-8$.

35. Bumpus JA (1989) Biodegradation of polycyclic hydrocarbons by Phanerochaete chrysosporium. Appl Environ Microbiol 55, 154-8.

36. Glenn JK, Gold MH (1983) Decolorization of several polymeric dyes by the lignin-degrading basidiomycete Phanerochaete chrysosporium. Appl Environ Microbiol 45, 1741-7.

37. Yadav JS, Reddy CA (1993) Degradation of benzene, toluene, ethylbenzene, and xylenes (BTEX) by the lignin-degrading basidiomycete Phanerochaete chrysosporium. Appl Environ Microbiol 59, 756-62.

38. Zheng Z, Obbard JP (2002) Polycyclic aromatic hydrocarbon removal from soil by surfactant solubilization and Phanerochaete chrysosporium oxidation. J Environ Qual 31, 1842-7.

39. Garon D, Krivobok S, Seigle-Murandi F (2000) Fungal degradation of fluorene. Chemosphere 40, 91-7.

40. Juhasz AL, Naidu R (2000) Bioremediation of high molecular weight polycyclic aromatic hydrocarbons: A review of the microbial degradation of benzo $[a]$ pyrene. Int Biodeter Biodegr 45, 57-88.

41. Lisowska K, Dlugonski J (1999) Removal of anthracene and phenanthrene by filamentous fungi capable of cortexolone 11-hydroxylation. J Basic Microbiol 39, 117-25.

42. Covino S, et al (2010) In vivo and in vitro polycyclic aromatic hydrocarbons degradation by Lentinus (Panus) tigrinus CBS 577.79. Bioresource Tech 101, 3004-12.

43. Covino S, et al (2010) An efficient PAH-degrading Lentinus (Panus) tigrinus strain: Effect of inoculum formulation and pollutant bioavailability in solid matrices. J Hazard Mater 183, 669-76.

44. Cerniglia CE (1993) Biodegradation of polycyclic aromatic hydrocarbons. Curr Opin Biotechnol 4, 331-8.

45. Sutherland JB (1992) Detoxification of polycyclic aromatic hydrocarbons by fungi. J Ind Microbiol Biotechnol 9, 53-61.

46. Tiehm A, Fritzsche C (1995) Utilisation of solubilised and crystalline mixtures of polycyclic aromatic hydrocarbons by a Mycobacterium sp. Appl Microbiol Biotechnol 42, 964-8.

47. Acevedo F, Pizzul L, Castillo MP, Cuevas R, Diez MC (2011) Degradation of polycyclic aromatic hydrocarbons by the Chilean white-rot fungus Anthracophyllum discolor. J Hazard Mater 185, 212-9.
48. Chigu NL, Hirosue S, Nakamura C, Teramoto H, Ichinose H, Wariishi H (2010) Cytochrome P450 monooxygenases involved in anthracene metabolism by the white-rot basidiomycete Phanerochaete chrysosporium. Appl Microbiol Biotechnol 87, 1907-16.

49. Ning D, Wang H, Ding C, Lu H (2010) Novel evidence of cytochrome P450-catalyzed oxidation of phenanthrene in Phanerochaete chrysosporium under ligninolytic conditions. Biodegradation 21, 889-901. 